\title{
Study on pyrethroid resistance of Brassicogethes aeneus F. (Coleoptera: Nitidulidae) in Hungary
}

\section{Zsolt MARCZALI - Balázs GOMBAI}

University of Pannonia, Georgikon Faculty, Institute of Plant Protection, Department of Applied Zoology, 8360 Keszthely, Deák F. u. 16. Hungary; E-mail: marczalizs@gmail.com

Keywords: winter oilseed rape, pollen beetle, pyrethroid resistance

\section{Introduction}

Pollen beetle (Brassicogethes aeneus $F$.) is one of the most serious pests of winter oilseed rape. Although it has been effectively controlled with pyrethroid insecticides for two decades, these insecticides have lost their efficacy. Their constant selective pressure has resulted in the occurrence and rapid spread of resistant pollen beetle populations (Slater et al., 2011, Thieme et al., 2008). The resistance of pollen beetles was first reported in 1999 in North-Eastern France (Zimmer \& Nauen, 2011, Veromann \& Toome, 2011), and until 2017, in the majority of European countries, pyrethroid resistant populations of pollen beetle dominate $(>60 \%$ are resistant). The objective of this study was to investigate the susceptibility or pyrethroid resistance of pollen beetle in different locations of Hungary.

\section{Materials and methods}

Pollen beetles were collected by sweep netting during the spring of 2016 at Keszthely and Enying. Approximately 100 to 200 adults were collected from each location across the infested fields. The beetles were kept in aerated plastic containers, with some dry paper towel at the bottom and some oil seed rape leaves and inflorescences as food source, to keep the vigour of the beetles until the laboratory tests. In the laboratory, a standard method recommended by Insecticide Resistance Action Committee (IRAC method No. 011) was used. During the tests, $20 \%$ and $100 \%$ of the typical field application rate of 7.5 g lambda-cyhalothrin as active ingredient per hectare were used.

\section{Results and discussion}

Table 1: Results of the laboratory tests with beetles collected at Enying

\begin{tabular}{|c|c|c|c|c|c|c|c|}
\hline Active ingredient & $\begin{array}{c}\text { Concentration } \\
\text { \% }\end{array}$ & Replicates & $\begin{array}{c}\text { Tested } \\
\text { adults } \\
\text { (pcs) }\end{array}$ & Dead & $\begin{array}{c}\text { Moribund } \\
\text { (M) }\end{array}$ & $\begin{array}{c}\text { Number } \\
\text { of reacting } \\
\text { adults } \\
\text { (D + M) }\end{array}$ & $\begin{array}{c}\text { Mortality } \\
\mathbf{\%}\end{array}$ \\
\hline lambda-cyhalothrin & $20 \%$ & 1 & 28 & 8 & 17 & 25 & $\mathbf{8 9 . 2 9}$ \\
\hline lambda-cyhalothrin & $20 \%$ & 2 & 39 & 10 & 6 & 16 & $\mathbf{4 1 . 0 3}$ \\
\hline lambda-cyhalothrin & $20 \%$ & 3 & 35 & 11 & 8 & 19 & $\mathbf{5 4 . 2 9}$ \\
\hline lambda-cyhalothrin & $100 \%$ & 1 & 23 & 6 & 7 & 13 & $\mathbf{5 6 . 5 0}$ \\
\hline lambda-cyhalothrin & $100 \%$ & 2 & 33 & 11 & 19 & 30 & $\mathbf{9 0 . 9 0}$ \\
\hline lambda-cyhalothrin & $100 \%$ & 3 & 31 & 10 & 17 & 27 & $\mathbf{8 7 . 1 0}$ \\
\hline Untreated/control & 0 & 1 & 30 & 0 & 0 & 0 & $\mathbf{0 . 0 0}$ \\
\hline Untreated/control & 0 & 2 & 39 & 0 & 0 & 0 & $\mathbf{0 . 0 0}$ \\
\hline Untreated/control & 0 & 3 & 35 & 0 & 0 & 0 & $\mathbf{0 . 0 0}$ \\
\hline
\end{tabular}


Table 2: Results of the laboratory tests with beetles collected at Keszthely

\begin{tabular}{|c|c|c|c|c|c|c|c|}
\hline Active ingredient & $\begin{array}{c}\text { Concentration } \\
\mathbf{\%}\end{array}$ & Replicates & $\begin{array}{c}\text { Tested } \\
\text { adults } \\
\text { (pcs) }\end{array}$ & $\begin{array}{c}\text { Dead } \\
\text { (D) }\end{array}$ & $\begin{array}{c}\text { Moribund } \\
\text { (M) }\end{array}$ & $\begin{array}{c}\text { Number } \\
\text { of reacting } \\
\text { adults } \\
\text { (D + M) }\end{array}$ & $\begin{array}{c}\text { Mortality } \\
\mathbf{\%}\end{array}$ \\
\hline lambda-cyhalothrin & $20 \%$ & 1 & 25 & 15 & 8 & 23 & $\mathbf{9 2 , 0 0}$ \\
\hline lambda-cyhalothrin & $20 \%$ & 2 & 26 & 13 & 9 & 22 & $\mathbf{8 4 , 6 2}$ \\
\hline lambda-cyhalothrin & $20 \%$ & 3 & 30 & 17 & 11 & 28 & $\mathbf{9 3 , 3 3}$ \\
\hline lambda-cyhalothrin & $100 \%$ & 1 & 34 & 28 & 6 & 34 & $\mathbf{1 0 0 , 0 0}$ \\
\hline lambda-cyhalothrin & $100 \%$ & 2 & 32 & 20 & 12 & 32 & $\mathbf{1 0 0 , 0 0}$ \\
\hline lambda-cyhalothrin & $100 \%$ & 3 & 35 & 24 & 11 & 35 & $\mathbf{1 0 0 , 0 0}$ \\
\hline Untreated/control & 0 & 1 & 30 & 0 & 0 & 0 & $\mathbf{0 , 0 0}$ \\
\hline Untreated/control & 0 & 2 & 36 & 0 & 0 & 0 & $\mathbf{0 , 0 0}$ \\
\hline Untreated/control & 0 & 3 & 34 & 0 & 0 & 0 & $\mathbf{0 , 0 0}$ \\
\hline
\end{tabular}

According to the evaluation and classification method of IRAC, the pollen beetle samples derived from Keszthely could be classified as susceptible (code 2), while the samples taken from Enying showed resistance (code 4).

\section{Conclusions}

Nowadays, constant monitoring of pollen beetle susceptibility to insecticides is essential for working out insecticide resistance management strategies, necessary for maintaining the effectiveness of chemical control.

\section{Acknowledgement}

This research was supported by the Hungarian Government and the European Union, with the co-funding of the European Regional Development Fund in the frame of Széchenyi 2020 Programme GINOP-2.3.2-15-2016-00054 project.

\section{References}

Nauen, R., Zimmer, C.T., Andrews, M., Slater, R., Bass, C., Ekbom, B., Gustafsson, G., Hansen, L.M., Kristensen, M., Zebitz, C.P. (2012): Target-site resistance to pyrethroids in European populations of pollen beetle, Meligethes aeneus F. Pesticide biochemistry and physiology, 103, 173-180. https://doi.org/10.1016/j. pestbp.2012.04.012

Slater, R., Ellis, S., Genay, J.P., Heimbach, U., Huart, G., Sarazin, M., Longhurst, C., Müller, A., Nauen, R., Rison, J.L., Robin, F. (2011): Pyrethroid resistance monitoring in European populations of pollen beetle (Meligethes spp.): a coordinated approach through the Insecticide Resistance Action Committee (IRAC). Pest Manag. Sci. 67: 633-638. https://doi.org/10.1002/ps.2101

Thieme, T., Drbal, U. Gloyna, K. Hoffmann, U. (2008): Different methods of monitoring susceptibility of oilseed rape beetles to insecticides. Bulletin OEPP/EPPO, 38, 114-117. https://doi.org/10.1111/j.13652338.2008.01193.x

Veromann, E., Toome, M. (2011): Pollen beetle (Meligethes aeneus Fab.) susceptibility to synthetic pyrethroids - Pilot study in Estonia. Agronomy Research, 9, 356-369.

Zimmer, C.T., Nauen, R. (2011): Cytochrome P450 mediated pyrethroid resistance in European populations of Meligethes aeneus (Coleoptera: Nitidulidae). Pesticide Biochemistry and Physiology, 100, 264-272. 